\title{
INVENTARIO PRELIMINAR DE, LA RIQUEZA GENÉRICA Y DISTRIBUCIÓN DE LOS COLEÓPTEROS ACUÁTICOS DEL DEPARTAMENTO DEL CHOCO, COLOMBIA
}

\section{PRELIMINARY INVENTORY OF THE GENERIC RICHNESS AND DISTRIBUTION OF THE AQUATIC COLEOPTERS OF THE CHOCÓ DEPARTMENT, COLOMBIA}

\author{
Zuleyma Mosquera Murillo ${ }^{1}$, Sandra Patricia Sánchez Vázquez² \\ ${ }^{1}$ Bióloga, M.Sc. Biología, Profesora Asistente, Programa de Biología. Universidad Tecnológica del Chocó. Ciudadela Uni- \\ versitaria, B/ Nicolás Medrano, Quibdó - Chocó, Colombia, e-mail: d-zuleyma.mosquera@utch.edu.co; ${ }^{2}$ Bióloga, Grupo de \\ Limnología. Universidad Tecnológica del Chocó. Ciudadela Universitaria, B/ Nicolás Medrano, Quibdó - Chocó, Colombia, \\ e-mail: sandrapatricia0220@gmail.com
}

\author{
Rev. U.D.C.A Act. \& Div. Cient. 21(1): 155-165, Enero-Junio, 2018 \\ https://doi.org/10.31910/rudca.v21.n1.2018.674
}

\section{RESUMEN}

Se presenta un registro preliminar sobre la riqueza y la distribución de los coleópteros acuáticos en el departamento del Chocó, a partir de 1.824 especímenes, entre larvas y adultos, que reposan en la Colección Limnológica del Chocó «CLCHInsec», de la Universidad Tecnológica del Chocó, recolectados en 20 municipios, a alturas entre 5 y 3.202 msnm, en 40 corrientes hídricas de diferente orden y 17 ecosistemas lénticos, de las cuencas de los ríos Atrato, San Juan y Baudó. Se reconocen 16 familias (4 Adephaga y 12 Polyphaga) y 40 géneros, con 4 familias y 20 géneros, que se registran por primera vez para el Chocó. La familia Psephenidae es la más abundante y más ampliamente distribuida, mientras que Hydraenidae es la de menor abundancia y distribución. La mayor riqueza taxonómica la posee los élmidos, con 12 géneros. Las rocas son el sustrato con mayor abundancia y riqueza específica. El mayor índice taxonómico se encuentra en la cuenca del río Atrato, con 16 familias y 33 géneros. Estos resultados permiten evidenciar la enorme diversidad de los coleópteros acuáticos y semiacuáticos en el departamento del Chocó y la necesidad de adelantar nuevas investigaciones, que amplíen su conocimiento taxonómico y ecológico en la región.

Palabras clave: Colección Limnológica, Coleoptera, ecosistemas acuáticos, Insecta.

\section{SUMMARY}

A preliminary record on the richness and distribution of aquatic Coleoptera in the Department of Chocó is presented.
The report is made from 1824 specimens (larvae and adults) found in the Limnological Collection of Chocó «CLCH-Insec» of the Technological University of Chocó, collected in 20 municipalities at altitudes between $5 \mathrm{~m}$ and $3202 \mathrm{~m}$, in 40 water streams of different order and 17 lentic ecosystems of the Atrato, San Juan and Baudó river basins. This collection includes 16 families (4 Adephaga and 12 Polyphaga) and 40 genera. Four families and 20 genera are register for Chocó for the first time. The family Psephenidae is the most abundant and most widely distributed, while Hydraenidae is of lowest abundance and distribution. The highest taxonomic richness is register for elmids with 12 genera. Rocks are the substrate with greatest abundance and specific richness. The largest taxonomic record occurs in the Atrato river basin with 16 families and 33 genera. These results show the enormous diversity of aquatic and semi - aquatic beetles in the department of Chocó and the need to carry out new research to increase their taxonomic and ecological knowledge in the region.

Key words: Limnological Collection, Coleoptera, aquatic ecosystems, Insecta.

\section{INTRODUCCIÓN}

Coleoptera es el orden más diverso de la clase Insecta, con aproximadamente 360.000 especies descritas (Beutel \& Pohl, 2006), de las cuales, existen cerca de 12.000 especies acuáticas. De las 170 familias descritas en el mundo, unas 30 tienen, en alguno de sus estadios, representantes acuáticos en la región neotropical (Miserendino \& Archangelsky, 2006). 
Los coleópteros acuáticos constituyen un grupo heterogéneo, que incluye taxa, pertenecientes a distintos linajes de los subórdenes Adephaga y Polyphaga. A nivel mundial es en los Adephaga, donde se puede evidenciar más claramente la evolución hacia el ambiente acuático, con una amplia radiación adaptativa y diversificación. Por su parte, los representantes del suborden Polyphaga constituyen, por el contrario, un grupo heterogéneo, ya que las familias que se han adaptado a vivir en ambientes acuáticos pertenecen a distintos linajes (Jerez \& Moroni, 2006).

Todos los cuerpos de aguas continentales constituyen hábitats favorables para los coleópteros acuáticos, donde forman parte de las comunidades lóticas y lénticas, gracias a las marcadas adaptaciones morfológicas y fisiológicas, que les facilitan colonizar diferentes ambientes acuáticos (Arias et al. 2007). Ecológicamente, estos insectos y principalmente su estado larvario forman parte de la fauna de macroinvertebrados bentónicos y participan en múltiples cadenas tróficas, donde actúan como depredadores, detritívoros, herbívoros o comedores de perifiton. También actúan como eslabones tróficos intermedios, entre productores primarios y consumidores secundarios, principalmente peces (Trémouilles et al. 1995; Archangelsky et al. 2009)

Constituyen un grupo muy útil para evaluar la biodiversidad, debido al gran número de especies que incluye, a que colonizan diferentes tipos de hábitats y a que su presencia en los ambientes acuáticos ocurre durante todo el año. Por lo dicho anteriormente, algunos investigadores los utilizan como indicadores de la calidad ecológica de los ecosistemas acuáticos (Sánchez-Fernández et al. 2006; Miserendino \& Archangelsky, 2006).

En Colombia, son importantes contribuciones al conocimiento de los coleópteros acuáticos los trabajos sobre ecología, riqueza, distribución y sobre todo evaluaciones de calidad de agua, con base en macroinvertebrados bentónicos, entre los que se destacan los de Montoya-Moreno et al. (2007), Arango et al. (2008), Posada-García et al. (2008), García et al. (2009), Chará-Serna et al. (2010; 2012), González-Gómez et al. (2012), Zúñiga et al. (2013). En el departamento del Chocó, los estudios sobre el orden son aun incipientes, reportándose, de forma fragmentaria, la presencia de 13 familias y 33 géneros (González et al. 2016; Mosquera-Murillo et al. 2016; Mosquera-Murillo \& Mosquera-Mosquera, 2017).

El presente artículo tiene como objetivo aportar información sobre la riqueza genérica y distribución del orden Coleoptera en el departamento del Chocó, a partir de la revisión de los especímenes, que se encuentran depositados en la Colección Limnológica del Chocó «CLCH-Insec».

\section{MATERIALES Y MÉTODOS}

La información taxonómica que se presenta es el resultado de la recopilación y de la revisión de información sobre larvas y adultos del orden Coleoptera, depositadas en la Colección Limnológica del Chocó «CLCH-Insec» (Universidad Tecnológica del Chocó), conservadas y preservadas en su totalidad, en alcohol al $80 \%$. Estos organismos fueron colectados, a través de proyectos de grado e investigaciones realizadas por el grupo de Limnología de la Universidad Tecnológica del Chocó, entre el 2002 y 2016, cuya información fue validada en todos los casos, para verificar los datos correspondientes a coordenadas, distribución altitudinal y procedencia, con el acompañamiento de personal del SiB-Colombia. Los especímenes de Coleoptera estudiados corresponden a estadios inmaduros y adultos colectados de forma cualitativa, en diferentes sustratos sumergidos, como rocas del lecho, hojarasca, troncos, vegetación ribereña, musgo y macrófitas; la determinación taxonómica, se realizó en el laboratorio de Limnología de la UTCH, para lo que se emplearon las claves de Fernández \& Domínguez (2001), Merrit et al. (2008) y Domínguez \& Fernández (2009), tanto para larvas como para los adultos, con la ayuda de un estereomicroscopio NIKON SMZ 745.

A partir de los datos obtenidos de la colección biológica, se elaboró un mapa con la representación geográfica de las áreas de distribución de orden Coleoptera en el departamento del Chocó (Figura 1), así como matrices por familias y géneros, con sus respectivas abundancias, distribución altitudinal, distribución por sustratos, cuencas y municipios del departamento.

\section{RESULTADOS Y DISCUSIÓN}

Riqueza faunística y abundancia relativa de coleópteros acuáticos. Los datos de los 1.824 ejemplares revisados (88,27\% adultos y $11,73 \%$ larvas) permiten reportar, de forma preliminar, para el departamento del Chocó, en los dos subórdenes, 16 familias y 40 géneros de coleópteros acuáticos (Tabla 1), los cuales, se encontraron asociados a 40 corrientes hídricas de diferente orden y 17 ecosistemas lénticos, de las cuencas de los ríos Atrato, San Juan y Baudó, ubicados en 20 municipios del departamento (Figura 1). Estos resultados constituyen un aporte muy significativo, que incrementa el número de taxones distribuidos en el departamento, puesto que el $25 \%$ de las familias y el $50 \%$ de los géneros son reportados por primera vez para el Chocó (Tabla 1).

De las 16 familias del orden Coleoptera registradas en la Colección Limnológica del Chocó, Psephenidae (Polyphaga), con el género Psephenus, es la que presenta el mayor nú- 


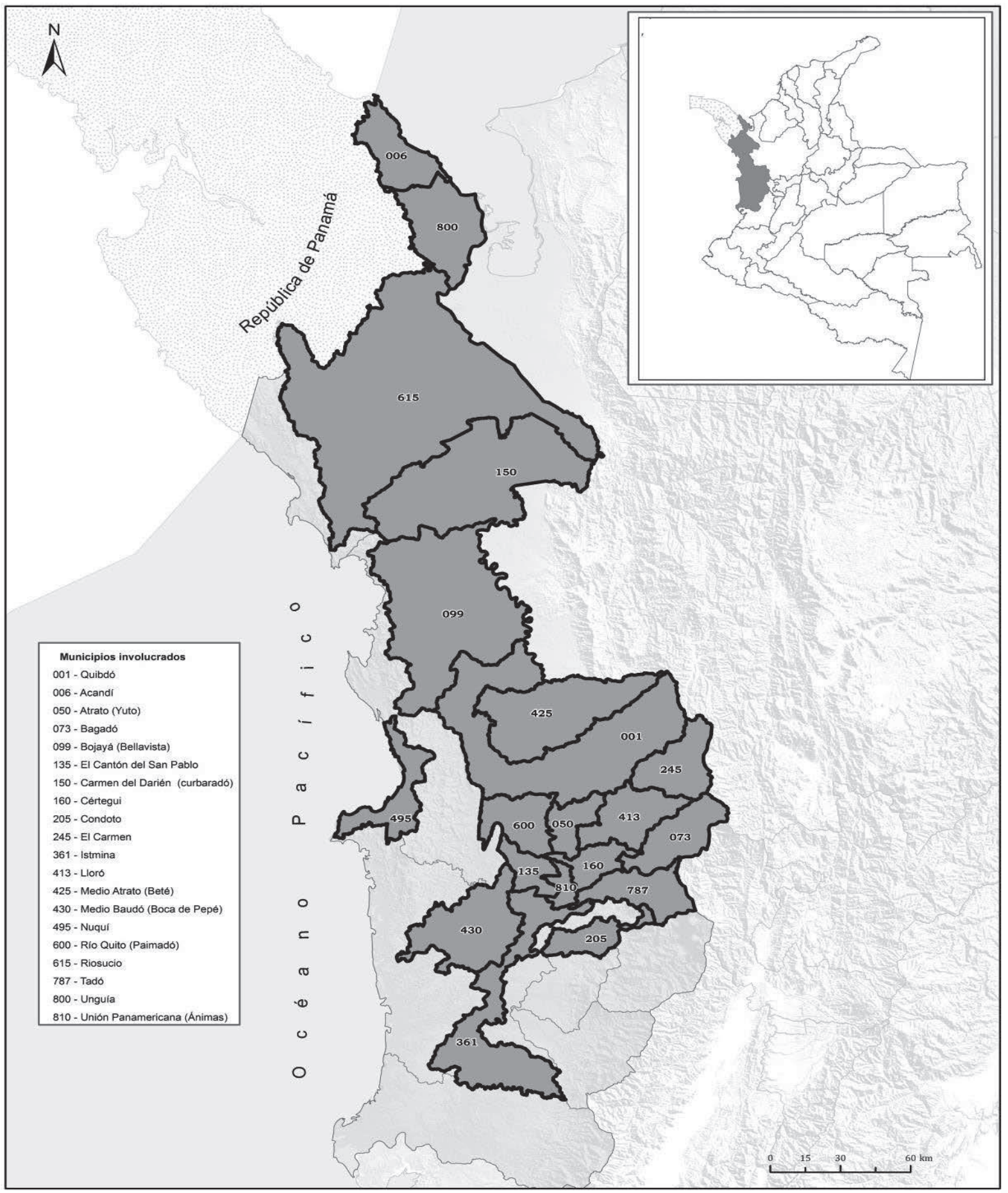

Figura 1. División política del departamento de Chocó (Colombia), con las áreas de distribución del orden Coleoptera, representadas en la colección en tono más oscuro.

mero de organismos, con el 36,84\%, seguida de Elmidae, con un $33,04 \%$ y Noteridae, con $12 \%$. Las restantes familias presentan abundancias inferiores al 5\%; sin embargo, Elmidae es la que muestra la mayor riqueza, con 12 géneros, seguida de Hydrophilidae, con 7 (Figura 2). La familia Psephenidae no se destaca por su abundancia en las investigaciones realizadas en otras regiones del país (Zúñiga et al. 2013; González-Gómez et al. 2012; Arias et al. 2007). Su 
Tabla 1. Listado taxonómico, abundancia relativa, etapa de vida y reporte de nuevos géneros del orden Coleoptera, para el departamento del Chocó, depositados en la Colección Limnológica del Chocó «CLCH-Insec».

\begin{tabular}{|c|c|c|c|}
\hline Taxón & $\begin{array}{l}\text { Abundancia } \\
\text { Relativa (\%) }\end{array}$ & Estadio & Registro \\
\hline \multicolumn{4}{|c|}{ Suborden Adephaga } \\
\hline \multicolumn{4}{|l|}{ Gyrinidae Latreille, 1810} \\
\hline Gyretes Brulle, 1835 & 2,58 & Larva, Adulto & \\
\hline Gyrinus Geoffroy, 1762 & 0,16 & Larva & \\
\hline Andogyrus Ochs, 1924 & 0,05 & Larva & \\
\hline Noteridae Thomson 1860 & & & Nuevo \\
\hline Hydrocanthus Say, 1823 & 12,01 & Larva, Adulto & Nuevo \\
\hline \multicolumn{4}{|l|}{ Dytiscidae Leach, 1815} \\
\hline Laccophilus Leach, 1815 & 2,91 & Larva, Adulto & \\
\hline Rhantus Dejean, 1833 & 0,77 & Larva, Adulto & Nuevo \\
\hline Copelatus Erichson, 1832 & 0,11 & Larva & Nuevo \\
\hline Carabidae Latreille, 1802 & & & Nuevo \\
\hline Dyschirius Bonelli, 1810 & 0,11 & Adulto & Nuevo \\
\hline Chlaenius Schrank, 1781 & 0,05 & Larva & Nuevo \\
\hline \multicolumn{4}{|c|}{ Suborden Polyphaga } \\
\hline \multicolumn{4}{|l|}{ Psephenidae Germar, 1818} \\
\hline Psephenus Haldeman, 1853 & 36,79 & Larva, Adulto & \\
\hline Acneus Horn, 1880 & 0,05 & Larva & Nuevo \\
\hline \multicolumn{4}{|l|}{ Elmidae Curtis, 1830} \\
\hline Cylloepus Erichson, 1847 & 5,92 & Larva, Adulto & \\
\hline Phanocerus Sharp, 1882 & 2,41 & Larva, Adulto & \\
\hline Hexanchorus Sharp, 1882 & 0,88 & Larva & \\
\hline Microcylloepus Hinton, 1935 & 0,71 & Larva, Adulto & \\
\hline Neocylloepus Brown, 1970 & 0,44 & Larva & \\
\hline Neoelmis Musgrave, 1935 & 1,75 & Larva, Adulto & \\
\hline Macrelmis Motschulsky, 1859 & 20,39 & Larva & \\
\hline Disersus Sharp, 1882 & 0,16 & Larva & \\
\hline Austrolimnius Carter \& Zeck, 1929 & 0,38 & Larva & \\
\hline Huleechius Brown, 1981 & 0,11 & Larva & \\
\hline Stenhelmoides Grouvelle, 1908 & 0,05 & Larva & \\
\hline Heterelmis Sharp, 1882 & 0,44 & Larva & \\
\hline \multicolumn{4}{|l|}{ Staphylinidae Latreille, 1802} \\
\hline Stenus Latreille, 1796 & 0,99 & Larva, Adulto & \\
\hline Pontamalota Casey, 1885 & 0,05 & Adulto & Nuevo \\
\hline \multicolumn{4}{|l|}{ Ptilodactylidae, Laporte 1836} \\
\hline Anchytarsus Guerin - Moneville, 1843 & 4,66 & Larva & \\
\hline \multicolumn{4}{|l|}{ Scirtidae Fleming, 1821} \\
\hline Scirtes Illiger, 1807 & 0,55 & Larva & \\
\hline Prionocyphon Redtenbacher, 1858 & 0,22 & Larva & Nuevo \\
\hline
\end{tabular}


Continuación Tabla 1.

\begin{tabular}{|c|c|c|c|}
\hline \multicolumn{4}{|l|}{ Hydrophilidae Latreille, 1802} \\
\hline Berosus Leach, 1817 & 0,05 & Adulto & Nuevo \\
\hline Helochares Mulsant, 1844 & 0,11 & Adulto & Nuevo \\
\hline Hydrophilus Geoffroy, 1762 & 0,27 & Larva, Adulto & \\
\hline Hydrochus Leach, 1817 & 0,71 & Larva & Nuevo \\
\hline Hydrobius Leach, 1815 & 0,05 & Larva & Nuevo \\
\hline Tropisternus Solier, 1834 & 0,71 & Larva & Nuevo \\
\hline Lampyridae Latreille, 1817 & 0,11 & Larva & Nuevo \\
\hline \multicolumn{4}{|l|}{ Curculionidae Latreille, 1802} \\
\hline Tanysphyrus Fabricius, 1792 & 0,22 & Larva & Nuevo \\
\hline \multicolumn{4}{|l|}{ Dryopidae Billberg, 1820} \\
\hline Helichus Erichson, 1847 & 0,16 & Larva, Adulto & \\
\hline Pelonomus Erichson, 1847 & 0,16 & Larva & Nuevo \\
\hline Lutrochidae Kasap \& Crowson, 1975 & & & Nuevo \\
\hline Lutrochus Erichson, 1847 & 1,37 & Larva & Nuevo \\
\hline \multicolumn{4}{|l|}{ Georissidae Laporte, 1819} \\
\hline Georissus Latreille, 1809 & 0,11 & Larva & \\
\hline \multicolumn{4}{|l|}{ Hydraenidae Mulsant,1844 } \\
\hline Hydraena Kugelann, 1794 & 0,05 & Adulto & \\
\hline
\end{tabular}

abundancia en la Colección podría estar asociada al buen estado de conservación de los ecosistemas representados en la misma, al tratarse de organismos considerados como importantes indicadores de calidad de agua y de estabilidad ambiental (Jerez \& Moroni, 2006; Archangelsky et al. 2009). Las formas inmaduras viven en ambientes acuáticos, donde, por lo general, son abundantes y se las halla sobre rocas, troncos, hojas y ramas, en las corrientes rápidas de ríos y de arroyos de agua clara y muy oxigenadas, con temperaturas bajas y estables (Lee \& Jach, 1995). Psephenus es un género muy común en corrientes de mayor tamaño, con sustratos pedregosos (Zúñiga et al. 2013), donde las larvas se localizan sobre o bajo las rocas, cantos y ramas sumergidos, mientras que los adultos lo hacen en las rocas y en la vegetación que se desarrolla sobre los márgenes de los ríos y de los arroyos (Arce-Pérez \& Shepard, 2001).

La riqueza de los élmidos que se observa en la Colección, coincide con González et al. (2016), quienes ubican a la región del Chocó biogeográfico, como una de las de mayor riqueza genérica para esta familia, en el país; igualmente, algunos aspectos de su biología y su ecología, como hábitos sedentarios y ser muy comunes en ambientes lóticos (Manzo, 2005), donde viven adheridos a una variedad de sustratos, como grava, rocas, raíces de árboles, hojas en descomposición, troncos, vegetación y musgos acuáticos (White \& Roughley, 2008; Gutiérrez-Fonseca, 2010), pueden explicar su riqueza en la región.
La familia Hydrophilidae es la segunda de mayor riqueza en la Colección (Figura 2), a pesar de su baja abundancia, que podría estar relacionada con aspectos biológicos de la misma, ya que, de acuerdo con García (2007), esta familia habita en mayor medida bajo las piedras o cualquier sustrato que se encuentre en el fondo de ecosistemas lénticos o adherida a la superficie arenosa de los bordes de dichos cuerpos de agua, lo que explica su baja abundancia en la colección, teniendo en cuenta que los sistemas lénticos representan solo el 29,82\%, de los referenciados en la misma.

Distribución de los coleópteros acuáticos. A nivel de las principales cuencas del departamento del Chocó, es la del río Atrato la que registra el mayor número de individuos de la Colección, con el 51,34\%, representados en 16 familias y 33 géneros; en segundo lugar, se encuentra la cuenca del río San Juan, con el $47,20 \%$, con 10 familias y 24 géneros y, en último lugar, la cuenca del río Baudó, con el $0,91 \%$, con 4 familias y 4 géneros. Cuatro familias y 4 géneros se registran en microcuencas pericontinentales, que fluyen directamente al océano Pacífico (Tabla 2).

La mayor abundancia y riqueza que se registra para el orden Coleoptera en el Atrato, se atribuye a que es el área más estudiada del departamento, dada su cercanía a centros poblados y facilidades de acceso; a diferencia del área del San Juan y en especial en el Baudó, que presentan problemas relacionados con infraestructura vial y orden público, que 


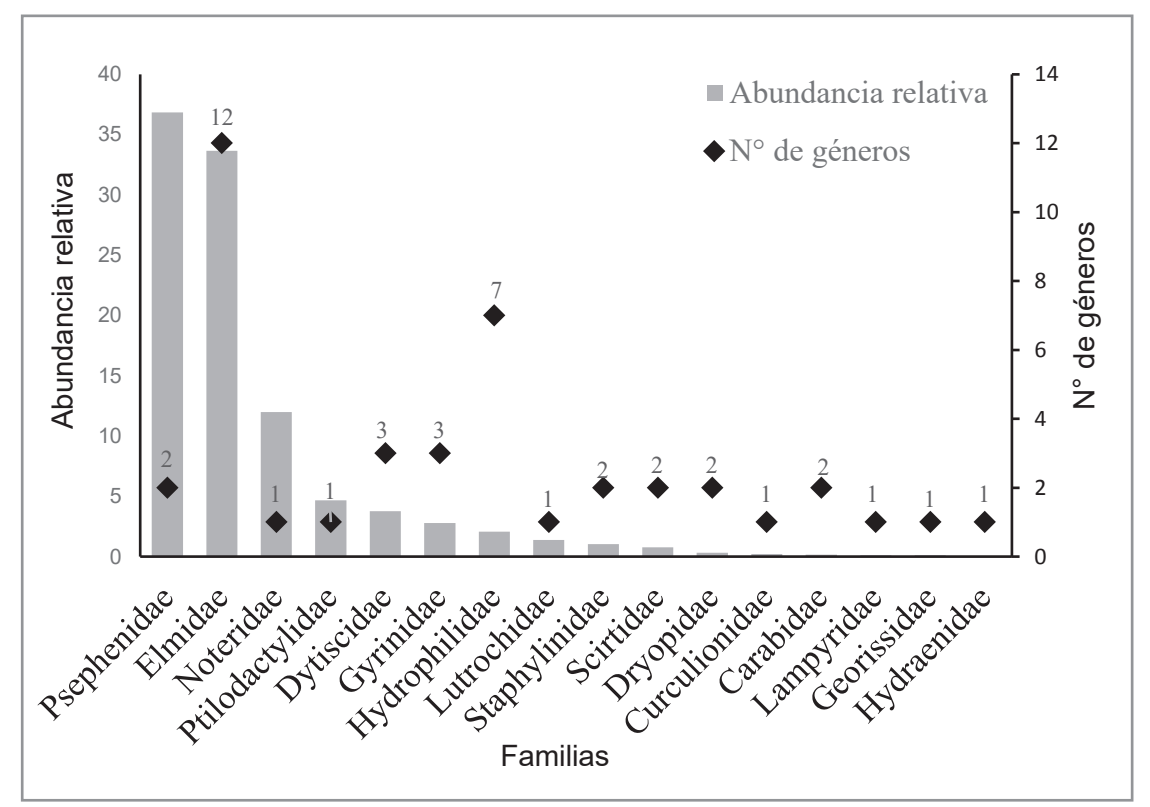

Figura 2. Abundancia relativa y riqueza genérica de familias de coleópteros acuáticos, registradas en la Colección Limnológica del Chocó (CLCH-Insec).

Tabla 2. Distribución de las familias y géneros de Coleoptera, en los municipios del Chocó, ubicación por Macrocuenca y rango de altura.

\begin{tabular}{|c|c|c|c|c|}
\hline Taxón & Distribución en el Chocó & Cuenca & Sustrato & $\begin{array}{l}\text { Rango de altura } \\
\text { (msnm) }\end{array}$ \\
\hline \multicolumn{5}{|l|}{ Gyrinidae } \\
\hline Gyretes & Qb, At, Up, Cdp, Ta, Mb & Atrato, San Juan, Baudó & $\mathrm{P}, \mathrm{H}, \mathrm{Vr}, \mathrm{Mu}$ & $44-132$ \\
\hline Gyrinus & Un, Is & Atrato, San Juan & $\mathrm{H}, \mathrm{Ma}$ & $5-133$ \\
\hline Andogyrus & Co & San Juan & $\mathrm{Vr}$ & 132 \\
\hline \multicolumn{5}{|l|}{ Noteridae } \\
\hline Hydrocanthus & Qb, At, Un, Rs, Bo, Cd, Ac & Atrato, Pacífica + & $\mathrm{P}, \mathrm{Vr}, \mathrm{Ma}$ & $5-72$ \\
\hline \multicolumn{5}{|l|}{ Dytiscidae } \\
\hline Laccophilus & $\begin{array}{l}\text { Qb, At, Un, Rs, Bo, Up, } \\
\text { Cdp, }\end{array}$ & Atrato, San Juan & $\mathrm{P}, \mathrm{H}, \mathrm{Vr}, \mathrm{Ma}$ & $5-110$ \\
\hline Rhantus & $\mathrm{Qb}, \mathrm{Ce}, \mathrm{Un}, \mathrm{Rs}, \mathrm{Ma}, \mathrm{Ac}$ & Atrato, Pacífica + & $\mathrm{H}, \mathrm{Ma}$ & $5-32$ \\
\hline Copelatus & $\mathrm{Cd}$ & Atrato & $\mathrm{Ma}$ & 14 \\
\hline \multicolumn{5}{|l|}{ Carabidae } \\
\hline Dyschirius & $\mathrm{Qb}$ & Atrato & $\mathrm{P}$ & 50 \\
\hline Chlaenius & $\mathrm{Ba}$ & Atrato & $\mathrm{P}$ & 3202 \\
\hline \multicolumn{5}{|l|}{ Psephenidae } \\
\hline Psephenus & $\begin{array}{l}\text { Qb, Ce, Llo, At, Ba, Is, Up, } \\
\text { Cdp, Ta, Co, Mb, Nu }\end{array}$ & Atrato, San Juan, Baudó, Pacífica + & $\mathrm{P}, \mathrm{H}, \mathrm{Vr}, \mathrm{T}$ & $32-3202$ \\
\hline Acneus & $\mathrm{Qb}$ & Atrato & $\mathrm{P}$ & 90 \\
\hline \multicolumn{5}{|l|}{ Elmidae } \\
\hline Cylloepus & $\begin{array}{l}\text { Qb, At, Ca, Is, Up, Cdp, } \\
\text { Ta, Co }\end{array}$ & Atrato, San Juan & $\mathrm{P}, \mathrm{H}$ & $25-2247$ \\
\hline
\end{tabular}




\begin{tabular}{|c|c|c|c|c|}
\hline Phanocerus & Qb, Up, Cdp, Ta, Nu & Atrato, San Juan & $\mathrm{P}, \mathrm{H}, \mathrm{Vr}, \mathrm{T}, \mathrm{Ma}$ & $32-138$ \\
\hline Hexanchorus & Qb, Llo, Un, Up & Atrato, San Juan & $\mathrm{P}, \mathrm{H}, \mathrm{Ma}$ & $5-103$ \\
\hline Microcylloepus & $\mathrm{Qb}, \mathrm{Ce}, \mathrm{Ca}, \mathrm{Is}, \mathrm{Ta}$ & Atrato, San Juan & $\mathrm{P}, \mathrm{H}$ & $37-2247$ \\
\hline Neocylloepus & $\mathrm{Qb}, \mathrm{At}, \mathrm{Ba}, \mathrm{Ta}$ & Atrato, San Juan & $\mathrm{P}$ & $90-3202$ \\
\hline Neoelmis & Qb, At, Cdp, Ta & Atrato, San Juan & $\mathrm{P}, \mathrm{H}, \mathrm{T}$ & $46-138$ \\
\hline Macrelmis & $\begin{array}{l}\text { Qb, Ce, At, Ca, Is, Up, } \\
\text { Cdp, Ta, Co }\end{array}$ & Atrato, San Juan & $\mathrm{P}, \mathrm{H}, \mathrm{Vr}$ & $37-2247$ \\
\hline Disersus & $\mathrm{Qb}, \mathrm{Ce}, \mathrm{Ta}$ & Atrato, San Juan & $\mathrm{P}, \mathrm{H}$ & $90-95$ \\
\hline Austrolimnius & $\mathrm{Ta}$ & San Juan & $\mathrm{H}$ & 95 \\
\hline Huleechius & At & Atrato & $\mathrm{P}$ & $60-72$ \\
\hline Stenhelmoides & $\mathrm{Mb}$ & Baudó & $\mathrm{Vr}$ & 32 \\
\hline Heterelmis & Qb, Rs, Ta, Co & Atrato, San Juan & $\mathrm{P}, \mathrm{H}, \mathrm{Ma}$ & $5-138$ \\
\hline \multicolumn{5}{|l|}{ Staphylinidae } \\
\hline Stenus & Qb, Rs, Up & Atrato, San Juan & $\mathrm{P}, \mathrm{H}, \mathrm{Ma}$ & $5-116$ \\
\hline Pontamalota & $\mathrm{Ta}$ & San Juan & $\mathrm{H}$ & 135 \\
\hline \multicolumn{5}{|l|}{ Ptilodactylidae } \\
\hline Anchytarsus & $\begin{array}{l}\text { Qb, Llo, At, Ca, Rq, Is, Up, } \\
\text { Ta, Mb, Nu }\end{array}$ & Atrato, San Juan, Baudó, Pacífica + & $\mathrm{P}, \mathrm{H}, \mathrm{Vr}$ & $34-2247$ \\
\hline \multicolumn{5}{|l|}{ Scirtidae } \\
\hline Scirtes & Qb, Un, Rs, Ma, Cdp & Atrato, San Juan & $\mathrm{P}, \mathrm{H}, \mathrm{T}, \mathrm{Ma}$ & $5-90$ \\
\hline Prionocyphon & $\mathrm{Qb}, \mathrm{Ba}, \mathrm{Cdp}$ & Atrato, San Juan & $\mathrm{P}, \mathrm{Vr}, \mathrm{T}$ & $46-3202$ \\
\hline \multicolumn{5}{|l|}{ Hydrophilidae } \\
\hline Berosus & $\mathrm{Qb}$ & Atrato & $\mathrm{P}$ & 50 \\
\hline Helochares & $\mathrm{Qb}$ & Atrato & $\mathrm{P}, \mathrm{Ma}$ & $32-90$ \\
\hline Hydrophilus & Un, Rs, Cd & Atrato & Ma & $5-27$ \\
\hline Hydrochus & Un, Rs, Bo & Atrato & $\mathrm{Ma}$ & $5-26$ \\
\hline Hydrobius & $\mathrm{Qb}$ & Atrato & $\mathrm{Mu}$ & 90 \\
\hline Tropisternus & Qb, Rs, Cd & Atrato & $\mathrm{Vr}, \mathrm{Ma}$ & $14-90$ \\
\hline Lampyridae & Qb, Ta & Atrato, San Juan & $\mathrm{P}$ & $46-132$ \\
\hline \multicolumn{5}{|l|}{ Curculionidae } \\
\hline Tanysphyrus & Rs, Cd & Atrato & Ma & $5-27$ \\
\hline \multicolumn{5}{|l|}{ Dryopidae } \\
\hline Helichus & $\mathrm{Ta}$ & San Juan & $\mathrm{P}, \mathrm{H}$ & $95-138$ \\
\hline Pelonomus & $\mathrm{Qb}$ & Atrato & $\mathrm{P}, \mathrm{H}$ & $56-90$ \\
\hline \multicolumn{5}{|l|}{ Lutrochidae } \\
\hline Lutrochus & $\mathrm{Qb}$ & Atrato & $\mathrm{P}$ & $46-96$ \\
\hline \multicolumn{5}{|l|}{ Georissidae } \\
\hline Georissus & Qb, Up & Atrato, San Juan & $\mathrm{P}, \mathrm{Mu}$ & $90-103$ \\
\hline \multicolumn{5}{|l|}{ Hydraenidae } \\
\hline Hydraena & $\mathrm{Qb}$ & Atrato & $\mathrm{P}$ & 46 \\
\hline
\end{tabular}

+ Microcuenca pericontinental.

Qb: Quibdó, Ce: Cértegui, At: Atrato, Llo: Lloro, Un: Unguía, Ba: Bagadó, Ca: Carmen de Atrato, Rs: Río Sucio, Bo: Bojaya, Cd: Carmen del Darién, Ma: Medio Atrato, Rq: Río Quito, Ta: Tadó, Up: Unión Panamericana, Is: Istmina, Co: Condoto, Cdp: Cantón de San Pablo, Mb: Medio Baudó, Nu: Nuquí, Ac: Acandí. P: piedra, H: hojarasca, T: tronco, Vr. vegetación ribereña, Ma: macrófitas, Mu: musgo. 
dificultan la realización de estudios sobre diferentes grupos biológicos, entre ellos, los insectos acuáticos, lo que explica su baja representación en la Colección.

Psephenidae es la familia mejor distribuida en la Colección Limnológica del Chocó, al encontrarse en el $60 \%$ de los municipios (12), seguida de Dytiscidae, con el 55\% (11), mientras que Lutrochidae e Hydraenidae, se registran en solo uno de los municipios representados en la misma (Tabla 2; Figura 3). La amplia distribución presentada por familias, como Psephenidae, Elmidae y Dytiscidae, confirma lo reportado para algunas zonas del Chocó, en investigaciones realizadas sobre macroinvertebrados acuáticos y calidad de agua, entre las que sobresalen las de Torres et al. (2006), Asprilla et al. (2006), Mosquera-Murillo \& Mosquera-Mosquera (2017). En particular, las familias Elmidae y Psephenidae viven casi exclusivamente en aguas corrientes, por sus altos requerimientos de oxígeno, mientras que los ditiscidos, a pesar de hallarse en casi todos los tipos de ambientes acuáticos continentales, prefieren las aguas lénticas, ya sean permanentes o temporarias (Archangelsky et al. 2009), siendo el grupo de coleópteros acuáticos más numeroso, diverso y mejor adaptado a la vida acuática, con todos sus estados de desarrollo acuáticos, excepto el de pupa (Nilsson, 2001).

Las familias Lutrochidae (Lutrochus) e Hydraenidae (Hydraena), se encontraron entre las de menor distribución. El género Lutrochus, hasta hace poco incluido dentro de la familia Limnichidae, actualmente, se le adjudica su propia familia (Archangelsky et al. 2009). Sus larvas son acuáticas y se encuentran en aguas lóticas y lénticas bien oxigenadas, entre la vegetación y los residuos vegetales (Roldán, 2003), donde se alimentan de algas y de materia orgánica (Domínguez \& Fernández, 2009). Los hidraenidos son coleópteros muy pequeños no nadadores, que viven en ambientes ribereños o asociados a la vegetación litoral (Archangelsky et al. 2009) y que se desplazan caminando sobre rocas y algas en las riberas de cuerpos de agua o en los márgenes de corrientes de aguas claras y fondo arenoso (Perkins, 1980).

Desde el punto de vista altitudinal, los géneros que presentaron los rangos más amplios fueron Psephenus (Psephenidae), Prionocyphon (Scirtidae), Neocylloepus y Cylloepus (Elmidae); sin embargo, para todos los géneros reportados, se amplía el rango de distribución altitudinal en el país, al registrarse en localidades por debajo de los $100 \mathrm{~m}$ (Tabla 2). Es importante destacar que 11 géneros $(27,5 \%)$ solo se registran en una de las localidades de estudio, por lo tanto, su valor en la distribución altitudinal fue puntual. Según Arias (2004), la altura sobre el nivel del mar es uno de los factores más definitivos en la abundancia y en la distribución de los coleópteros acuáticos, debido a que existen géneros con amplio rango de distribución y otros son característicos de un piso altitudinal en particular; asimismo, Marchant et al. (1995) han sugerido que la altitud afecta indirectamente la distribución de los macroinvertebrados acuáticos, debido a los cambios en temperatura del agua y contenido de oxígeno.

Los ejemplares revisados del orden Coleoptera, se encontraron asociados a seis sustratos: piedras del lecho, hojarasca, troncos, vegetación ribereña, musgo y macrófitas, siendo

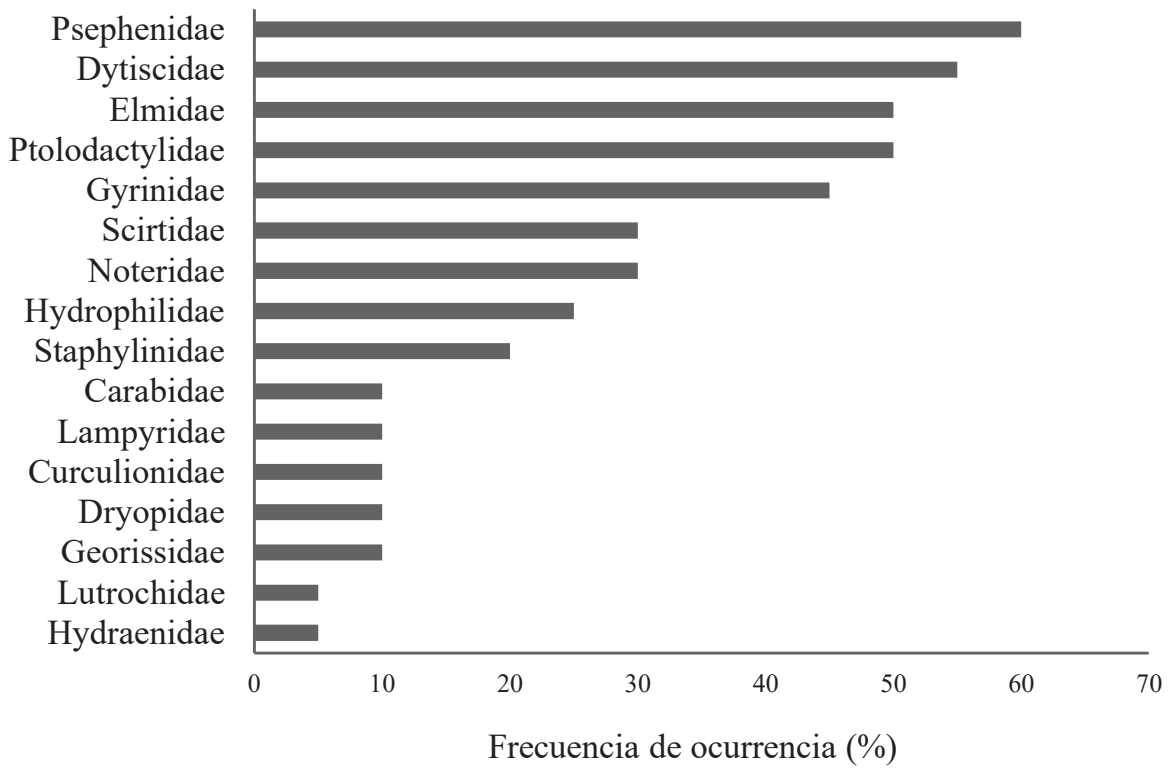

Figura 3. Frecuencia de ocurrencia de familias de Coleoptera en 20 municipios del departamento del Chocó - Colombia. 
piedras del lecho el que registra la mayor riqueza de géneros (28), seguido de hojarasca (20), macrófitas (15), vegetación ribereña (11), troncos (5) y finalmente musgo (3) (Tabla 2). Estos resultados pueden estar asociados, en el caso de los sistemas lóticos, a la mayor estabilidad, oferta alimenticia y refugio, que ofrecen las piedras y la hojarasca a los coleópteros acuáticos, mientras que para los sistemas lénticos, las macrófitas brindan una importante fuente de alimento y refugio para algunas familias de coleópteros (Rúa-García, 2015).

Estos resultados constituyen un importante aporte al conocimiento de la riqueza genérica y de distribución de los coleópteros acuáticos y semiacuáticos en el departamento del Chocó y en el país, poniendo, además en evidencia, la enorme diversidad del orden en la región y la necesidad de adelantar nuevas investigaciones, que involucren aspectos, tanto taxonómicos como biológicos, a nivel de familias, géneros y especies, sobre todo en zonas poco exploradas del departamento.

Agradecimientos. Las autoras agradecen al laboratorio de Limnología, a la Vicerrectoría de Investigaciones y al Centro de Investigación en Biodiversidad y Hábitat "CEIBHA", de la Universidad Tecnológica del Chocó, por su apoyo para la organización y la creación de la Colección Limnológica del Chocó, a partir de la cual, se generó la información que se presenta en este artículo. A las biólogas Karen E. Córdoba y Yasiris Salas Tovar (QEPD) y a la estudiante Karen Palomeque, por su participación en la organización de la colección y trabajo de laboratorio. A la profesora María del Carmen Zúñiga y a Marcela González-Córdoba, de la Universidad del Valle, por su apoyo en la identificación de los élmidos. Agradecemos, igualmente, a los revisores anónimos por sus comentarios y sugerencias, quienes contribuyeron a mejorar la versión final del manuscrito. Conflictos de intereses: El manuscrito fue preparado y revisado con la participación de todos los autores, quienes declaramos que no existe conflicto de intereses, que ponga en riesgo la validez de los resultados presentados.

\section{BIBLIOGRAFÍA}

1. ARANGO, M.C.; ÁlVAREZ-ARANGO, L.F.; ARANGO, G.A.; TORRES, O.E; MONSALVE, A. DE J. 2008. Calidad del agua de las quebradas La Cristalina y La Risaralda, San Luis, Antioquia. Rev. Escuela Ingeniería de Antioquia. 9:121-141.

2. ARCE-PÉREZ, R.; SHEPARD, W. 2001. Sinopsis de la familia Psephenidae (Coleoptera: Dryopoidea) de Norte y Centroamérica. Folia Entomológica Mexicana. 40(3):397-406.
3. ARCHANGELSKY, M.; MANZO, V.; MICHAT, M.C.; TORRES, P.L.M. 2009. Coleoptera. Domínguez, E.; Fernández, H.R. (eds.), Macroinvertebrados bentónicos sudamericanos. Sistemática y biología. Vol. 2, Fundación Miguel Lillo, Tucumán, p.411-468.

4. ARIAS, D. 2004. Diversidad de coleópteros acuáticos en la cuenca del río Coello. Ibagué, Colombia: Universidad del Tolima. 78p.

5. ARIAS-DÍAZ, D.M.; REINOSO, G.; GUEVARA-C., G.; VILLA-NAVARRO, F. 2007. Distribución espacial y temporal de los coleópteros acuáticos de la cuenca del río Coello (Tolima, Colombia). Caldasia. 29:177-194.

6. ASPRILLA, S.; MOSQUERA, Z.; RIVAS, M. 2006. Macroinvertebrados acuáticos como indicadores de calidad ecológica del agua en la parte media del río Cabí (Quibdó - Chocó). Rev. Asoc. Colom. Cien. Biol. 18:43-50.

7. BEUTEL, R.G.; POHL, H. 2006. Endopterygote systematic where do we stand and what is the goal (Hexapoda, Arthropoda) Systematic Entomology. 31:202219.

8. CHARÁ-SERNA, A.M.; CHARÁ, J.D.; ZÚĨIIGA, M. DEL C.; PEDRAZA, G.X.; GIRALDO, L.P. 2010. Clasificación trófica de insectos acuáticos en ocho quebradas protegidas de la ecorregión cafetera colombiana. Universitas Scientiarum. 15(1):27-36.

9. CHARÁ-SERNA, A.M.; CHARÁ, J.D.; ZÚĨIIGA, M. DEL C.; PEARSON, R.G.; BOYERO, L. 2012. Diets of leaf litter-associated invertebrates in three tropical streams. Annales de Limnologie. 48:139-144.

10. DOMÍNGUEZ, E.; FERNÁNDEZ, H.R. (Ed.). 2009. Macroinvertebrados Bentónicos Sudamericanos. Fundación Miguel Lillo, Tucumán, Argentina. 656p.

11. FERNÁNDEZ, H.; DOMÍNGUEZ, E. 2001. Guía para la determinación de los artrópodos bentónicos sudamericanos. Universidad Nacional de Tucumán. Facultad de Ciencias Naturales e Instituto M. Lillo, Tucumán. 282p.

12. GARCÍA, M. 2007. Coleópteros Acuáticos (Insecta: Coleoptera) del Estado Apure, Venezuela. Bol. Centro Investigaciones Biológicas. 41:255-264. 
13. GARCÍA, J.; CANTERA, J.; ZÚÑIIGA, M. DEL C.; MONTOYA, Y. 2009. Estructura y diversidad de las comunidades de macroinvertebrados acuáticos en la cuenca baja del río Dagua (Andén pacífico vallecaucano - Colombia). Rev. Ciencias, Facultad de Ciencias Naturales y Exactas. 13:25-48.

14. GONZÁLEZ-CÓRDOBA, M.; ZÚĨIIGA, M. DEL C.; MOSQUERA, Z.; SÁNCHEZ, S. 2016. Riqueza y distribución de Elmidae (Insecta: Coleoptera: Byrrhoidea) en el departamento del Chocó, Colombia. Intropica. 11:85-95.

15. GONZÁLEZ-GÓMEZ, S.M.; RAMÍREZ, Y.P.; MEZA- SALAZAR, A.M.; GOMES-DIAS, L. 2012. Diversidad de macroinvertebrados acuáticos y calidad de agua de quebradas abastecedoras del municipio de Manizales. Bol. Científico Centro Museos Museo de Historia Natural. 16(2):135-148.

16. GUTIÉRREZ-FONSECA, P.E. 2010. Guía ilustrada para el estudio ecológico y taxonómico de los insectos acuáticos del Orden Coleoptera en El Salvador. En: Springer, M.; \& Sermeño Chicas,.M. (eds.). Formulación de una guía metodológica estandarizada para determinar la calidad ambiental de las aguas de los ríos de El Salvador, utilizando insectos acuáticos. Proyecto Universidad del Salvador-Organización de los Estados Americanos (OEA). Editorial Universitaria UES, San Salvador, El Salvador. 64p.

17. JEREZ, V.; MORONI, J. 2006. Diversidad de coleópteros acuáticos en Chile. Gayana. 70(1):72-81.

18. LEE, C.F.; JACH, M.A. 1995. Psephenidae l. Checklist of the Psephenidae of China (Coleoptera). Water Beetles of China. 1:349-354.

19. MANZO, V. 2005. Key to the South America genera of Elmidae (Insecta: Coleoptera) with distributional data. Studies on Neotropical Fauna and Environment. 40(3):201-208.

20. MARCHANT, R.; BARMUTA, L.A.; CHESSMAN, B.C. 1995. Preliminary study of the ordination and classification of macroinvertebrate communities from running waters in Victoria, Australia. Aust. Mar. Freshwater Res. 45:945-962.

21. MERRITT, R.W.; CUMMINS, K.W.; BERG, M.B. 2008. An Introduction to the Aquatic Insects of North America. Dubuque, Kendall/Hunt. Publishing Company. 1214p.
22. MISERENDINO, M.L.; ARCHANGELSKY, M. 2006. Aquatic Coleoptera distribution and environmental relationships in a large Patagonian river. International Review of Hydrobiology. 91(5):423-437.

23. MONTOYA-MORENO, Y.; ACOSTA-GARCÍA, Y.; ZULUAGA-ZULUAGA, E.; GARCÍA, A. 2007. Evaluación de la biodiversidad de insectos acuáticos y de calidad fisicoquímica y biológica del Río Negro (AntioquiaColombia). Rev. Universidad Católica de Oriente. 23:70-87.

24. MOSQUERA-MURILLO, Z.; GÓMEZ, M.A.; GONZÁLEZ, M.B. 2016. Diversidad de macroinvertebrados acuáticos asociados con Bromeliaceae en una zona de bosque pluvial tropical, Chocó, Colombia. Rev. Biodivers. Neotrop. 6(2):147-153.

25. MOSQUERA-MURILLO, Z.; MOSQUERA-MOSQUERA, M.M. 2017. Diversidad de la entomofauna acuática y calidad de agua en quebradas del rio San Juan, Chocó - Colombia. Rev. U.D.C.A Act. \& Div. Cient. 20(1):149-161.

26. NILSSON, A.N. 2001. Word catalogue of insects. Vol. 3. Dytiscidae (Coleoptera). Apollo Books, Stenstrup, Denmark. 395p.

27. PERKINS, P.D. 1980. Aquatic beetles of the family Hydraenidae in the Western Hemisphere: Classification, Biogeography and inferred Phylogeny (Insecta: Coleoptera). Quaestiones Entomologicae. 16:1-554.

28. POSADA-GARCÍA, J.A.; ABRIL-RAMÍREZ, G.; PARRASÁNCHEZ, L.N. 2008. Diversidad de los macroinvertebrados acuáticos del páramo de Frontino (Antioquia, Colombia). Caldasia. 30 (2):441-455.

29. ROLDÁN, G.A. 2003. Bioindicación de la calidad del agua en Colombia: Uso del método BMWP/Col. Universidad de Antioquia. Colombia. 170p.

30. RÚA-GARCÍA, G. 2015. Macroinvertebrados acuáticos asociados a raíces de Eichhornia crassipes (Mart) Solms, en la ciénaga de Zapayán, Magdalena-Colombia. Intropica. 10:52-59.

31. SÁNCHEZ-FERNÁNDEZ, D.; ABELLÁN, P.; MELLADO, A.; VELASCO, J.; MILLÁN, A. 2006. Are water beetles good indicators of biodiversity in Mediterranean aquatic ecosystems? The case of the Segura river basin (SE Spain). Biodiversity Conservation. 15:4507-4520. 
32. TORRES, Y.; ROLDAN, G.; ASPRILLA, S.; RIVAS, T. 2006. Estudio preliminar de algunos aspectos ambientales y ecológicos de las comunidades de peces y macroinvertebrados acuáticos en el río Tutunendo, Chocó-Colombia. Rev. Acad. Colomb. Cienc. Ex. Fis. Nat. 114(30):67-76.

33. TRÉMOUILLES, E.R. 1995. Dytiscidae: Methlinae - Hydroporinae. En: Z. A. de Castellanos, (Dir.). Fauna de Agua Dulce de la República Argentina, 37 (1), FECIC, Buenos Aires, p.1-82.

34. WHITE, D.S.; ROUGHLEY, R.E. 2008. Aquatic Coleoptera. Pegs. 571-671. In: Merritt, R.W.; Cummins, K.W.;
Berg, M.B. (eds.). An Introduction to the Aquatic Insects of North America. Dubuque, Kendall/Hunt. Publishing Company. 1214p.

35. ZÚĨIIGA, M. DEL C.; CHARÁ, J.; GIRALDO, L.P.; CHARÁ, A.M.; PEDRAZA, G.X. 2013. Composición de la comunidad de macroinvertebrados acuáticos en pequeñas quebradas de la región Andina colombiana, con énfasis en la entomofauna. Dugesiana. 20(2):263-277.

Recibido: Octubre 18 de 2017

Aceptado: Febrero 15 de 2018

\section{Cómo citar:}

Mosquera Murillo, Z.; Sánchez Vázquez, S.P. 2018. Riqueza genérica y distribución de los coleópteros acuáticos del Departamento del Chocó - Colombia, depositados en la Colección Limnológica de la Universidad Tecnológica del Chocó. Rev. U.D.C.A Act. \& Div. Cient. 21(1): 155-165. 